\title{
Qualidade da água na área de entorno do antigo lixão de Tangará da Serra (MT)
}

O descarte irregular de resíduos sólidos em áreas de disposição inadequada constitui uma realidade no Brasil, gerando impactos ao meio ambiente (no solo e nas águas subterrâneas e superficiais) e risco à saúde pública. Nesse sentido, o objetivo desta pesquisa foi avaliar a qualidade das águas subterrâneas e superficial adjacentes do antigo lixão de Tangará da Serra-MT. A metodologia consistiu em realizar análises físicas, químicas e microbiológicas das águas subterrâneas e superficial no período chuvoso e seco compreendidos entre setembro de 2019 e junho de 2020. Foram avaliados quatro poços de monitoramento (P1, P2, P3 e P4) com distância de 100 a 2.000 metros do lixão. A avaliação da água superficial ocorreu no Córrego do Meio, (P5) próximo ao lixão (nascente) e a (P6) a jusante. Foram realizados a avaliação da qualidade da água utilizando o índice de Qualidade da Água (IQA). Os resultados obtidos nos poços de
monitoramento mostraram maiores valores nos parâmetros (cor real, cor aparente, condutividade elétrica, nitrito, nitrato, fósforo, dureza total, demanda bioquímica de oxigênio (DBO) e demanda química de oxigênio (DQO)) no período de seca, em todos os poços, evidenciando que com a redução do volume de água na seca ocorre um aumento da concentração. Nas águas superficiais os dados de nitrato, resíduos totais e Escherichia coli, demostraram maior valores à jusante, em função da vegetação alterada por ações antrópica e do carreamento de partículas na área da nascente verificou-se a melhor qualidade das águas devido a conservação da vegetação na área. Por consequência, os valores de (IQA) do Córrego do Meio classificaram as águas da nascente (P5) como boas e as da jusante (P6) como regular. Os resultados obtidos inferem não haver carreamento de poluentes do antigo lixão capaz de impactar na qualidade das águas subterrâneas e superficial para os parâmetros estudados.

\section{Water quality in the surrounding area of the old dump of Tangará da Serra (MT)}

\begin{abstract}
The irregular disposal of solid waste in areas of inadequate disposal is a reality in Brazil, generating acts to the environment (on public soil and in underground and surface waters) and health risk. In this sense, the objective of this research was to evaluate the quality of the groundwater and adjacent surface waters of the old dump of Tangará da Serra-MT. The methodology consisted of carrying out physical, substance and microbiological analyzes of ground and surface water in the rainy and dry period between September 2019 and June 2020 . Four monitoring wells (P1, P2, P3 and P4) were taken with a distance of 100 the 2,000 meters from the dump. The assessment of surface water occurred in Córrego do Meio, (P5) close to the dump (source) and (P6) downstream. Water quality assessment was carried out using the Water Quality Index (WQI). The results obtained in the monitoring wells decrease the higher values in the and (P6) downstream. Water quality assessment was carried out using the Water Quality Index (WQI). The results obtained in the monitoring wells decrease the higher values in the
parameters (real color, apparent color, electrical conductivity, nitrite, nitrate, phosphorus, total hardness, biochemical oxygen demand (BOD) and chemical oxygen demand (COD)) in the period drought, in all wells, showing that with the reduction of water volume in drought an increase in concentration occurs. In surface waters, the data of nitrate, total residues and Escherichia coli, showed higher values downstream, due to the alteration altered by anthropic actions and the transport of particles while in the spring area it was verified the best water quality due to the conservation of vegetation in the area. Consequently, the values of (IQA) of the Córrego do Meio classified as spring waters (P5) as good and those of downstream (P6) as regular. The results obtained infer that there is no carrying of pollutants from the old dump capable of impacting the quality of groundwater and surface water.
\end{abstract}

Keywords: Water resources; Groundwater; Surface water; Environmental impact.

Topic: Engenharia Ambiental

Reviewed anonymously in the process of blind peer.
Received: 04/03/2021

Approved: 24/03/2021
Luanna Mênithen Souza Silva Santos (iD) Universidade Federal de Mato Grosso, Brasil http://lattes.cnpq.br/6454314942616700 http://orcid.org/0000-0002-5160-5275 Imenithen@gmail.com

Nayra Nalessa de Campos Monteiro (iD) Universidade Federal de Mato Grosso, Brasil http://lattes.cnpq.br/0216754874406213 http://orcid.org/0000-0002-2390-3777

nayracmonteiro@gmail.com

Gabriel Figueiredo de Moraes (iD)

Universidade Federal de Mato Grosso, Brasil http://lattes.cnpq.br/8101837260214809 http://orcid.org/0000-0002-5137-1421 gabriel.moraes4@gmail.com
Wilhan Douglas dos Reis (iD)

Universidade Federal de Mato Grosso, Brasil http://lattes.cnpq.br/3340400763795544 http://orcid.org/0000-0002-6039-9733

eng.wilhan@gmail.com

\section{Aldecy de Almeida Santos (iD)}

Universidade Federal de Mato Grosso, Brasi http://lattes.cnpq.br/3224921282419849 http://orcid.org/0000-0003-4361-307X

aldecy_allmeida@yahoo.com.br
Referencing this:

SANTOS, L. M. S. S.; MONTEIRO, N. N. C.; MORAES, G. F.; REIS, W. D.; SANTOS, A. A.. Qualidade da água na área de entorno do antigo lixão de Tangará da Serra (MT). Revista Ibero Americana de Ciências Ambientais, v.12, n.3, p.323-332, 2021. DOI: http://doi.org/10.6008/CBPC2179-6858.2021.003.0027 


\section{INTRODUÇÃO}

Nos países em desenvolvimento, a gestão de resíduos sólidos tornou-se progressivamente mais desafiadora. Fatores como o crescimento exponencial e desordenado da população humana, atrelada ao aumento da produção industrial e ao desenvolvimento constante de novas tecnologias, aceleram a produção de resíduos não só em quantidade, mas também na composição do resíduo gerado (MISHRA et al., 2019).

O resíduo urbano, ou seja, os resíduos sólidos urbanos (RSU) quando acumulado e iniciado o processo de degradação biológica, produz um líquido denominado lixiviado. Com a produção do lixiviado, esses poluentes podem percolar e infiltrar contaminando o solo, as águas subterrâneas e superficiais, alterando a qualidade da água de áreas próximas aos lixões, apresentando riscos à saúde e ao meio ambiente, diminuindo a qualidade de vida das populações no entorno (KAPELEWSKA et al., 2019).

Entre 2010 e 2014 houve um crescimento do número de lixões no Brasil e nos anos consecutivos, alcançando 1.297 unidades cadastradas, sendo que no ano de 2017 ainda se encontravam cadastrados 1.091 áreas de lixões no Brasil, conforme dados do Sistema Nacional de Informações sobre Saneamento (SNIS), (BRASIL, 2017b). De acordo com o panorama de 2018 da Associação Brasileira de Empresas de Limpeza Pública e Resíduos Especiais, o Brasil ainda possui 2.976 lixões em operação (ABRELPE, 2018).

O controle e avaliação dos parâmetros físicos, químicos e microbiológicos das águas (superficial e subterrânea) em um lixão desativado é de grande importância, pois pode identificar a potencial poluidor de lixiviados e percolados (KAPELEWSKA et al., 2019).

No município de Tangará da Serra - MT, o antigo lixão de encontra-se situado no divisor de águas das duas microbacias hidrográficas do córrego Ararão e Bezerra Vermelho (MELO et al., 2019). Nesta perspectiva, esta pesquisa buscou realizar um diagnóstico das águas subterrâneas e superficial do entorno do lixão desativado em 2004 deste município. Para tanto, buscou-se avaliar a qualidade física e química das águas subterrâneas e superficial (Córrego do Meio) do entorno do antigo lixão.

\section{MATERIAIS E MÉTODOS}

\section{Área de estudo}

O município de Tangará da Serra, está localizado na porção sudoeste do Estado de Mato Grosso entre as coordenadas Latitude UTM $8383699.83 \mathrm{mS}$ e Longitude $447496.38 \mathrm{mE}$, estando a uma altitude de 427 metros. Possui uma área total de $11.601,104 \mathrm{~km}^{2}$, com uma área urbana de 58,3 km² (TANGARÁ DA SERRA, 2020). O município dispõe de uma população de 83.431 mil habitantes sendo 75.921 mil habitantes na área urbana (IBGE, 2011).

A localização do antigo lixão da cidade de Tangará da Serra está distante $4 \mathrm{~km}$ do centro da cidade (Figura 1). O clima da região de acordo com Köppen é o tropical úmido megatérmico (Aw) ou seja, quente semiúmido. Segundo Dallacort et al. (2010), os valores médios anuais de temperatura são de 24,4 ㄴ C, com precipitação média anual variando de 1.300 a 2000 mm e umidade relativa do ar variando entre 70 e $80 \%$. A estação chuvosa está concentrada no período de setembro a abril, e entre maio e agosto estabelece-se a 
estação seca (CRUZ et al., 2008).

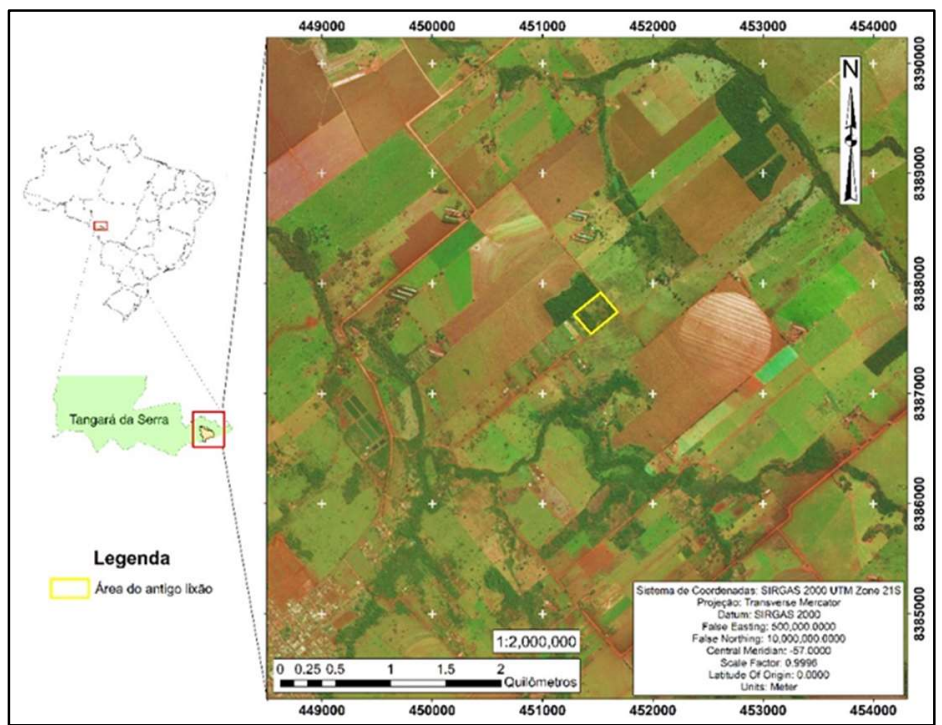

Figura 1: Localização do antigo lixão da cidade de Tangará da Serra-MT.

As classes de solo encontradas na região são latossolo vermelho escuro distrófico, latossolo vermelho distroférrico, latossolo vermelho eutroférrico e argissolo vermelho-amarelo distrófico, predominando o argissolo vermelho (BRASIL, 1997; BEHLING, 2009). A vegetação está inserida entre mata de transição de floresta amazônica e cerrado, apresentando matas densas nas encostas, no alto da Serra de Tapirapuã e cerrado no alto da Serra dos Parecis (GOUVEIA, 2013; BEHLING, 2009; CRUZ et al., 2008).

O antigo lixão municipal possui uma área superficial de aproximadamente 7,3 hectares (TANGARÁ DA SERRA, 2020) e localiza-se entre latitude $8387726.45 \mathrm{mS}$ e longitude $451477.57 \mathrm{mE}$ estando inserida no divisor de águas, entre as microbacias do córrego Ararão e Bezerra Vermelho sendo tributários do rio Sepotuba. Esta área foi utilizada para disposição de resíduos até o ano de 2004.

\section{Coleta e análise das águas subterrâneas e superficial}

Na Tabela 1 apresenta as descrições e características dos locais de amostragem das águas subterrâneas e superficiais.

Tabela 1: Pontos de amostragem de água subterrânea e superficial do antigo Lixão de Tangará da Serra.

\begin{tabular}{|c|c|c|c|c|c|}
\hline Ponto & Cota $(\mathrm{m})$ & UTM & Profundidade $(\mathrm{m})$ & Distância do lixão (m) & Tipo de Ponto \\
\hline P1 & 334 & $\begin{array}{l}451672.81 \mathrm{~m} \mathrm{E} \\
8389597.59 \mathrm{~m} \mathrm{~S}\end{array}$ & 8,37 & 1.870 & Poço raso \\
\hline P2 & 375 & $\begin{array}{l}451598.72 \mathrm{~m} \mathrm{E} \\
8387969.13 \mathrm{~m} \mathrm{~S}\end{array}$ & 11,40 & 214 & Poço raso \\
\hline P3 & 381 & $\begin{array}{l}451281.74 \mathrm{~m} \mathrm{E} \\
8387712.42 \mathrm{~m} \mathrm{~S}\end{array}$ & 50,00 & 183 & Poço profundo \\
\hline P4 & 365 & $\begin{array}{l}451808.31 \mathrm{~m} \mathrm{E} \\
8387571.01 \mathrm{~m} \mathrm{~S}\end{array}$ & 14,50 & 365 & Poço raso \\
\hline P5 & 344 & $\begin{array}{l}451954.17 \mathrm{~m} \mathrm{E} \\
8388491.07 \mathrm{~m} \mathrm{~S}\end{array}$ & ---- & 878 & Água superficial \\
\hline P6 & 330 & $\begin{array}{l}451554.10 \mathrm{~m} \mathrm{E} \\
8389620.29 \mathrm{~m} \mathrm{~S}\end{array}$ & ---- & 1.900 & Água superficial \\
\hline
\end{tabular}

As amostras de água subterrânea foram coletadas em quatro poços, sendo três do tipo cisterna (P1, P2 e P4) e um poço do tipo profundo (50m) (P3) localizados nas áreas no entorno do lixão. Nos pontos de 
água subterrâneas P2 e P4, por não possuírem estrutura de bombas hidráulicas para sucção da água dos poços, foi necessário utilizar coletores de água descartável do tipo Geobailer. Nos outros pontos P1 e P3 a coleta foi realizada diretamente na torneira dos poços.

As amostras de água superficial foram coletadas em córrego efêmero, tendo sido nesta pesquisa classificado como Classe 2 em ambiente lêntico, conforme CONAMA no. 357 (CONAMA, 2005), sendo as amostragens ocorridas no córrego do Meio. Foram realizadas coletas na nascente designado P5 e outro a jusante cerca $1.200 \mathrm{~m}$ designado P6.

As metodologias utilizadas para as determinações dos parâmetros físicos e químicos estão apresentadas nas Tabela 2, 3 e 4 respectivamente, as unidades usuais, assim como os limites estabelecidos pelas normativas Portaria MS de Consolidação no. 5 (BRASIL, 2017a) e com a Resolução CONAMA no. 396 (CONAMA, 2008), CONAMA no. 357 (CONAMA, 2005) e as metodologias estabelecidas pelo APHA (2012).

Tabela 2: Parâmetros físicos analisados, limites estabelecidos e metodologias analíticas. Limites: Consolidação MS no․ 5/2017 e CONAMA no. 396/2008.

\begin{tabular}{llll}
\hline Parâmetros & Unidades & Limites & Metodologia Analítica / APHA (2012) \\
\hline Cor real & $\mathrm{mg} \mathrm{Pt}-C o ~ L^{-1}$ & --- & Espectrofotometria / 2120 C \\
Cor aparente & ${\mathrm{mg} \mathrm{Pt}-\mathrm{Co} \mathrm{L}^{-1}}^{--}$ & 15,0 & Espectrofotometria / 2120 C \\
Condutividade elétrica & $\mathrm{\mu S} \mathrm{cm}-1$ & --- & Potenciometria / NBR 14340:1999 / 2510 A \\
Turbidez & $\mathrm{NTU}$ & 5,0 & Método nefelométrico / 2130 B \\
Sólidos totais & $\mathrm{mg} \mathrm{L}^{-1}$ & 1.000 & Gravimétrico / NBR 10664:1989 / 2540 B \\
Temperatura & ${ }^{\circ} \mathrm{C}$ & ---- & Multiparâmetro / 2550 B \\
\hline
\end{tabular}

Tabela 3: Parâmetros químicos analisados e metodologias analíticas. Limites: Consolidação MS no. 5/2017 e CONAMA no. 396/2008.

\begin{tabular}{lll}
\hline Parâmetros & Unidades & Metodologia Analítica - APHA (2012) \\
\hline $\mathrm{pH}$ & - & Multiparâmetro, potenciometria / NBR 7353:2014 / 4500 H+B \\
$\mathrm{DBO}_{5,20}$ & $\mathrm{mg} \mathrm{O}_{2} \mathrm{~L}^{-1}$ & Incubação à 20 ${ }^{\circ} \mathrm{C}$ e 5 dias/NBR 12614:1992 / 5210 B \\
$\mathrm{DQO}$ & $\mathrm{mg} \mathrm{O} \mathrm{L}^{-1}$ & Refluxo fechado / NBR 10357:1998 / 5220 D \\
Nitrito & ${\mathrm{mg} \mathrm{N}-\mathrm{NO}_{2} \mathrm{~L}^{-1}}^{\text {Nitrato }}$ & Espectrofotometria / NBR 13796:1997 / 4500 \\
Fósforo total & ${\mathrm{mg} \mathrm{N}-\mathrm{NO}_{3} \mathrm{~L}^{-1}}^{\mathrm{mg} \mathrm{P}-\mathrm{PO}_{4}^{-3} \mathrm{~L}^{-1}}$ & Espectrofotometria / NBR 13796:1997 / 4500 \\
Dureza & $\mathrm{mg} \mathrm{CaCO}_{3} \mathrm{~L}^{-1}$ & Espectrofotometria / NBR 12772:1992 / 4500 PE \\
\hline
\end{tabular}

Tabela 4: Parâmetros físicos, químicos e microbiológico analisados e metodologias analíticas. Limites: CONAMA no. 357/2005 para Classe 2.

\begin{tabular}{|c|c|c|}
\hline Parâmetros & Unidades & Metodologia Analítica - APHA (2012) \\
\hline $\mathrm{pH}$ & - & Multiparâmetro, potenciometria / NBR 7353:2014 / $4500 \mathrm{H}^{+} \mathrm{B}$ \\
\hline Temperatura & ${ }^{\circ} \mathrm{C}$ & Multiparâmetro / 2550 B \\
\hline Turbidez & NTU & Método nefelométrico / 2130 B \\
\hline Sólidos totais & $\mathrm{mg} \mathrm{L}^{-1}$ & Gravimétrico / BR 10664:1989 / 2540 B \\
\hline $\mathrm{DBO}_{5,20}$ & $\mathrm{mg} \mathrm{O}_{2} \mathrm{~L}^{-1}$ & Incubação à $20^{\circ} \mathrm{C}$ e 5 dias/NBR 12614:1992 / 5210 B \\
\hline Oxigênio dissolvido & $\mathrm{mg} \mathrm{O}_{2} \mathrm{~L}^{-1}$ & Multiparâmetro, potenciometria \\
\hline Nitrato & $\mathrm{mg} \mathrm{N}-\mathrm{NO}_{3} \mathrm{~L}^{-1}$ & Espectrofotometria / NBR 13796:1997 / 4500 \\
\hline Fósforo total & $\mathrm{mg} \mathrm{P}-\mathrm{PO}_{4}{ }^{-3} \mathrm{~L}^{-1}$ & Espectrofotometria / NBR 12772:1992 / 4500 PE \\
\hline Escherichia coli & NMP $100 \mathrm{~mL}^{-1}$ & Colilert \\
\hline
\end{tabular}

Foram realizados monitoramentos nos pontos de coleta de água subterrânea e superficial no período chuvoso nos meses de setembro a dezembro de 2019 e fevereiro de 2020 e no período de seca nos meses de maio a julho de 2020.

A qualidade da água superficial do córrego do Meio foi classificada e expressa de acordo com o Índice de Qualidade da Água (IQA). Foram considerados nove parâmetros, com ênfase ao biológico $E$. coli, além de $\mathrm{pH}$, nitrogênio e fósforo total, sólidos totais, temperatura, turbidez, oxigênio dissolvido e Demanda 
Bioquímica de Oxigênio $\left(\mathrm{DBO}_{5,20}\right)$.

A equação utilizada para a determinação do IQA é:

$$
\sum_{i=1 W_{i}=1}^{n}
$$

Onde:

IQA: Índice de Qualidade da Água, um número entre 0 e 100;

Wi: peso correspondente ao i-ésimo parâmetro, atribuído em função da sua importância para a conformação global

de qualidade, sendo que o somatório dos mesmos deve atingir 1 ; qi: qualidade do i-ésimo parâmetro, um número entre 0 e 100, obtido da respectiva curva média de variação de qualidade; $\mathrm{e}$ n: número de parâmetros do índice.

Os pesos (w), foram fixados para cada parâmetro em função da sua importância para a conformação global da qualidade da água, conforme apresentado na Tabela 5 e estabelecido pela CETESB.

Tabela 5: Parâmetros físicos, químicos e microbiológicos e seus respectivos pesos para o cálculo IQA-CETESB.

\begin{tabular}{lc}
\hline Parâmetro & Peso \\
\hline OD $(\%$ de saturação) & 0,17 \\
Escherichia coli (NMP/100 mL) & 0,15 \\
$\mathrm{pH}$ & 0,12 \\
$\mathrm{DBO}_{5,20}(\mathrm{mg} / \mathrm{L})$ & 0,10 \\
Nitrato $(\mathrm{mg} / \mathrm{L})$ & 0,10 \\
Fósforo total $(\mathrm{mg} / \mathrm{L})$ & 0,10 \\
Temperatura $\left({ }^{\circ} \mathrm{C}\right)$ & 0,10 \\
Turbidez (UNT) & 0,08 \\
Sólidos Totais (mg/L) & 0,08 \\
\hline
\end{tabular}

\section{RESULTADOS E DISCUSSÃO}

\section{Água subterrânea}

Os resultados das análises físico-químicas obtidos para as amostras de água subterrâneas coletadas nos poços monitorados no entorno do antigo lixão de Tangará da Serra-MT estão apresentados na Tabelas 6 e 7, assim como as unidades usuais, com valores médios e desvio padrão. Em relação ao poço P1, todas as amostras apresentaram valores de $\mathrm{pH}$ marginalmente inferior a 6,0 para o período seco, encontrando-se fora da faixa estabelecidas pelas normativas vigentes. Verifica-se que, com exceção dos valores de pH encontrados em P1 período da seca, os demais valores para P1, P2, P3 e P4 encontram-se no limite estabelecido pelas normativas vigentes.

Tabela 6: Resultados dos parâmetros físico-químicos das águas subterrâneas nos poços 1 e 2, nos períodos chuvoso e seco monitoradas no entorno do antigo Lixão de Tangará da Serra-MT.

\begin{tabular}{|c|c|c|c|c|c|c|c|c|c|}
\hline \multirow{2}{*}{ Parâmetros } & \multirow{2}{*}{ Unidade } & \multicolumn{2}{|c|}{ P1 Chuva } & \multicolumn{2}{|l|}{ P1 Seca } & \multicolumn{2}{|c|}{ P2 Chuva } & \multicolumn{2}{|l|}{ P2 Seca } \\
\hline & & M. & D.P. & M. & D.P. & M. & D.P. & M. & D.P. \\
\hline $\mathrm{pH}$ & ---- & 5,91 & 0,43 & 5,57 & 0,26 & 6,59 & 0,50 & 6,48 & 0,09 \\
\hline Condutividade & $\mu \mathrm{S} . \mathrm{cm}^{-1}$ & 31,32 & 5,79 & 31,60 & 6,07 & 136,43 & 10,74 & 142,67 & 4,21 \\
\hline Cor real & mg Pt-Co L-1 & 0,53 & 0,30 & 0,68 & 0,44 & 0,43 & 0,25 & 0,99 & 0,11 \\
\hline Cor aparente & $\mathrm{mg}$ Pt-Co L-1 & 2,26 & 0,53 & 4,17 & 1,26 & 4,40 & 1,97 & 6,17 & 1,89 \\
\hline Turbidez & NTU & 0,42 & 0,07 & 0,88 & 0,43 & 1,65 & 0,98 & 2,50 & 0,51 \\
\hline Sólidos totais & $m g L^{-1}$ & 5,33 & 0,58 & 7,10 & 2,04 & 22,0 & 10,44 & 65,67 & 11,93 \\
\hline Nitrito & $\mathrm{mg} \mathrm{N}-\mathrm{NO}_{2}-\mathrm{L}^{-1}$ & 0,0046 & 0,0010 & 0,0072 & 0,0010 & 0,0082 & 0,0008 & 0,0099 & 0,0035 \\
\hline Nitrato & $\mathrm{mg} \mathrm{N}-\mathrm{NO}_{3}{ }^{-} \mathrm{L}^{-1}$ & 0,046 & 0,021 & 0,074 & 0,017 & 0,083 & 0,021 & 0,117 & 0,031 \\
\hline Fósforo & $\mathrm{mg} \mathrm{P}-\mathrm{PO}_{4} \mathrm{~L}^{-1}$ & 0,025 & 0,008 & 0,036 & 0,018 & 0,062 & 0,029 & 0,082 & 0,036 \\
\hline Dureza & $\mathrm{mg} \mathrm{CaCO}_{3} \mathrm{~L}^{-1}$ & 0 & 0 & 0 & 0 & 118,3 & 18,1 & 145,0 & 32,5 \\
\hline DQO & $\mathrm{mg} \mathrm{O}_{2} \mathrm{~L}^{-1}$ & 0,60 & 0,55 & 1,67 & 0,58 & 1,00 & 1,00 & 3,00 & 1,73 \\
\hline $\mathrm{DBO}_{5,20}$ & $\mathrm{mg} \mathrm{O}_{2} \mathrm{~L}^{-1}$ & 0,60 & 0,55 & 0,60 & 0,55 & 0,67 & 0,58 & 1,08 & 0,14 \\
\hline
\end{tabular}

(M.): média e (D.P.): desvio padrão. 
De acordo com Feitosa et al. (2008) os valores de pH para P1, P2, P3 e P4 estão próximo da faixa características para águas subterrâneas com pH entre (5,5 e 8,5), não apresentaram diferença significativa entre os períodos chuvoso e seco para os valores de $\mathrm{pH}$ e condutividades elétrica. Observa-se que as maiores concentrações foram encontradas em P3, poço mais profundo, dados justificados pela maior solubilização dos sais presentes na rocha no período da pesquisa.

Tabela 7: Resultados dos parâmetros físico-químicos das águas subterrâneas nos poços 3 e 4, nos períodos chuvoso e seco monitoradas no entorno do antigo Lixão de Tangará da Serra-MT.

\begin{tabular}{|c|c|c|c|c|c|c|c|c|c|}
\hline \multirow{2}{*}{ Parâmetros } & \multirow{2}{*}{ Unidade } & \multicolumn{2}{|c|}{ P3 Chuva } & \multicolumn{2}{|l|}{ P3 Seca } & \multicolumn{2}{|c|}{ P4 Chuva } & \multicolumn{2}{|l|}{ P4 Seca } \\
\hline & & M. & D.P. & M. & D.P. & M. & D.P. & M. & D.P. \\
\hline $\mathrm{pH}$ & ---- & 7,26 & 0,31 & 7,33 & 0,25 & 6,23 & 0,32 & 6,53 & 0,05 \\
\hline Condutividade & $\mu \mathrm{S} \mathrm{cm} \mathrm{cm}^{-1}$ & 177,00 & 14,90 & 180,57 & 4,39 & 33,32 & 9,33 & 43,39 & 16,87 \\
\hline Cor real & mg Pt-Co L-1 & 0,39 & 0,34 & 0,56 & 0,27 & 0,76 & 0,06 & 1,20 & 0,20 \\
\hline Cor aparente & mg Pt-Co L-1 & 1,78 & 0,77 & 7,00 & 4,58 & 4,04 & 0,80 & 8,60 & 1,01 \\
\hline Turbidez & NTU & 0,67 & 0,04 & 0,76 & 0,18 & 3,50 & 1,05 & 4,99 & 1,85 \\
\hline Sólidos totais & $\mathrm{mg} \mathrm{L}^{-1}$ & 22,40 & 4,72 & 82,00 & 14,80 & 7,33 & 1,11 & 21,20 & 10,28 \\
\hline Nitrito & $\mathrm{mg} \mathrm{N}-\mathrm{NO}_{2}-\mathrm{L}^{-1}$ & 0,0055 & 0,0021 & 0,0083 & 0,0009 & 0,0089 & 0,0019 & 0,0114 & 0,0032 \\
\hline Nitrato & $\mathrm{mg} \mathrm{N}-\mathrm{NO}_{3}{ }^{-} \mathrm{L}^{-1}$ & 0,056 & 0,033 & 0,085 & 0,015 & 0,084 & 0,042 & 0,154 & 0,040 \\
\hline Fósforo & $\mathrm{mg} \mathrm{P}-\mathrm{PO}_{4} \mathrm{~L}^{-1}$ & 0,041 & 0,026 & 0,057 & 0,016 & 0,078 & 0,023 & 0,102 & 0,021 \\
\hline Dureza & $\mathrm{mg} \mathrm{CaCO}_{3} \mathrm{~L}^{-1}$ & 206,00 & 65,25 & 315,00 & 107,73 & 1,20 & 2,68 & 0 & 0 \\
\hline DQO & $\mathrm{mg} \mathrm{O}_{2} \mathrm{~L}^{-1}$ & 0,60 & 0,55 & 1,67 & 0,58 & 0,70 & 0,67 & 3,00 & 1,00 \\
\hline $\mathrm{DBO}_{5,20}$ & $\mathrm{mg} \mathrm{O}_{2} \mathrm{~L}^{-1}$ & 0,61 & 0,38 & 0,54 & 0,50 & 0 & 0 & 0,67 & 0,58 \\
\hline
\end{tabular}

(M.): média e (D.P.): desvio padrão.

As concentrações obtidas para cor real e aparente não apresentaram diferenças significativas para P1, P2 e P3, apresentaram valores mínimos e máximos variando entre 0,05 e 1,10 mg Pt-Co L-1, para cor real, entre 1,01 e 8,06 mg Pt-Co L-1, para aparente, respectivamente para os períodos chuvoso e seco. Enquanto P4 variou entre 0,70 e 1,40 mg Pt-Co L-1 para cor real e 3,24 e 9,61 mg Pt-Co $\mathrm{L}^{-1}$ para aparente, respectivamente para os períodos chuvoso e seco. Os resultados obtidos da cor nos poços monitorados, identificou concentrações menor no período chuvoso, sugerindo que, com o aumento da precipitação, ocorre diluição dos compostos responsáveis pela coloração na água. A Portaria MS de Consolidação no. 5 (BRASIL, 2017a) determina somente que as concentrações não ultrapassem 15 mg Pt-Co L ${ }^{-1}$ para cor aparente, não sendo estabelecido parâmetro para cor real.

As concentrações obtidas para turbidez não apresentaram diferenças significativas para os pontos avaliados, sendo que P1, P2 e P3 apresentaram valores mínimos e máximos entre 0,35 e 3,01 NTU, respectivamente, para os períodos chuvoso e seco. Enquanto P4 variou entre 2,45 e 6,84 NTU, respectivamente para os períodos chuvoso e seco. A maiores concentrações observadas para P4, tanto para cor como para turbidez, deve-se as características construtivas de poço raso.

Não foi determinada diferença significativa em P1 para os períodos analisados, sendo que as concentrações de sólidos totais variaram entre 4,75 e 9,14 mg L-1, enquanto P2, P3 e P4 apresentaram diferença significativa entre os períodos chuvoso e seco, sendo as concentrações de 6,22 e $96,80 \mathrm{mg} \mathrm{L}^{-1}$ para os pontos, sendo o mínimo observado para P4 na chuva e P3 na seca, sendo todas as maiores concentrações de P2, P3 e P4 na seca. Os resultados corroboram com os dados levantados para condutividade elétrica.

Em relação ao nitrito o valor o P1 apresentou diferença significativa em função de obras de aprofundamento do poço para o aumento da captação de água realizado no período de seca. Para as 
concentrações de nitrito em P2, P3 e P4, não houve diferença significativa observada, com concentrações de nitrito entre 0,0034 a 0,014 mg L $\mathrm{NO}_{2}^{-1}$, onde o mínimo foi observado em P3 no período chuvoso e a concentração máxima em P4 no período seco.

Para nitrato, não houve diferença significativa P1, P2, P3 e P4, sendo que as concentrações variaram entre 0,023 e $0,194 \mathrm{mg} \mathrm{L}^{-1} \mathrm{NO}_{3}^{-}$para os períodos de chuva e seca, respectivamente, com a menor concentração do período de chuva em P3 e a maior concentração na seca em P4, com as maiores concentrações médias no período de seca.

Para o nitrito e nitrato, não foram determinadas concentrações superiores ao estabelecido pelas normativas, sendo que as concentrações permissíveis para a resolução CONAMA no. 396 (CONAMA, 2008) e para a Portaria MS de Consolidação no. 5 (BRASIL, 2017a) que determinam a concentração máxima de 1,0 $\mathrm{mg} \mathrm{L}^{-1}$ para nitrito e $10,0 \mathrm{mg} \mathrm{L}^{-1}$ para nitrato.

As concentrações de fósforo nas amostras de água subterrânea não apresentaram diferenças significativas entre os períodos nas concentrações para P1, P2, P3 e P4, onde foi observado mínimas e máximas entre 0,017 a $0,123 \mathrm{mg} \mathrm{L}^{-1} \mathrm{PO}_{4}^{-3}$, respectivamente para os períodos chuvoso e seco, onde as maiores concentrações se apresentaram na seca. As menores e as maiores concentrações no período de seca foram observadas em P1 e P4, variando entre 0,018 a 0,123 mg L-1 PO-34. A Resolução CONAMA no. 396 (CONAMA, 2008) e a Portaria MS de Consolidação no. 5 (BRASIL, 2017a) não estabelecem padrões estabelecidos ou recomendados para as concentrações fósforo.

As concentrações de dureza total para P1, P2, P3 e P4 se apresentaram inferiores ao valor máximo permitido pelas normativas em todos os pontos amostrados na pesquisa, sendo que a Portaria MS de Consolidação no. 5 (BRASIL, 2017a), determina uma concentração máxima de dureza total de $500 \mathrm{mg} \mathrm{L}^{-1}$ de $\mathrm{CaCO}_{3}$.

As concentrações obtidas para $\mathrm{DQO}$ e $\mathrm{DBO}_{5,20}$ não apresentaram diferenças significativas para P1, P2 e P3 durante os períodos de chuva e seca. Os poços apresentaram concentrações mínimas e máximas variando entre 0,05 e $4,73 \mathrm{mg} \mathrm{O}_{2} \mathrm{~L}^{-1}$ e 0 e $1,22 \mathrm{mg} \mathrm{O}_{2} \mathrm{~L}^{-1}$ para DQO e $\mathrm{DBO}_{5,20}$, respectivamente, para os períodos chuvoso e seco, sendo as maiores médias observadas em P2. Enquanto P4 apresentou as menores médias no período chuvoso e as maiores no seco, variando 0,70 e $4,00 \mathrm{mg} \mathrm{O}_{2} \mathrm{~L}^{-1}$ e 0 e $1,25 \mathrm{mg} \mathrm{O}_{2} \mathrm{~L}^{-1}$ para $\mathrm{DQO}$ e $\mathrm{DBO}_{5,20}$, respectivamente, tendo apresentado diferença significativas entre os períodos avaliados.

De acordo com Feitosa et al. (2008), as concentrações características para águas subterrâneas apresentam DQO na faixa de 1 a $5 \mathrm{mg} \mathrm{O}_{2} \mathrm{~L}^{-1}$ e $\mathrm{DBO}_{5,20}$, em geral são inferiores a $1,0 \mathrm{mg} \mathrm{O}_{2} \mathrm{~L}^{-1}$. A Resolução CONAMA no. 396 (CONAMA, 2008) e a Portaria MS de Consolidação no. 5 (BRASIL, 2017a) não estabelecem padrões estabelecidos ou recomendados para os parâmetros de $\mathrm{DQO}$ e $\mathrm{DBO}_{5,20}$.

\section{Água superficial}

Os resultados das análises físico-químicas obtidos para as amostras de água superficiais coletadas nos pontos P5 e P6 monitorados no Córrego do Meio adjacentes ao antigo Lixão de Tangará da Serra-MT estão apresentados na Tabelas 8, assim como as unidades usuais, com valores médios e desvio padrão. 
Tabela 8: Resultados dos parâmetros físico-químicos das águas superficiais no Córrego do Meio, nos períodos chuvoso e seco monitoradas nas adjacências do antigo Lixão de Tangará da Serra-MT.

\begin{tabular}{|c|c|c|c|c|c|c|c|c|c|}
\hline \multirow{2}{*}{ Parâmetros } & \multirow{2}{*}{ Unidade } & \multicolumn{2}{|c|}{ P5 Chuva } & \multicolumn{2}{|c|}{ P5 Seca } & \multicolumn{2}{|c|}{ P6 Chuva } & \multicolumn{2}{|c|}{ P6 Seca } \\
\hline & & M. & D.P. & M. & D.P. & M. & D.P. & M. & D.P. \\
\hline $\mathrm{pH}$ & ---- & 6,45 & 0,12 & 6,37 & 0,21 & 6,48 & 0,29 & 6,50 & 0,10 \\
\hline Temperatura & ${ }^{\circ} \mathrm{C}$ & 26,0 & 1,2 & 24,8 & 1,7 & 27,5 & 1,34 & 24,6 & 1,97 \\
\hline Turbidez & NTU & 8,15 & 5,00 & 2,10 & 1,20 & 17,29 & 8,34 & 8,60 & 2,70 \\
\hline Resíduo total & $\mathrm{mg} \mathrm{L}^{-1}$ & 22,50 & 18,04 & 42,20 & 1,70 & 30,33 & 8,89 & 46,50 & 7,77 \\
\hline $\mathrm{DBO}_{5,20}$ & $\mathrm{mg} \mathrm{O}_{2} \mathrm{~L}^{-1}$ & 3,50 & 2,38 & 4,80 & 2,80 & 2,83 & 0,98 & 4,90 & 1,40 \\
\hline Oxigênio dissolvido & $\mathrm{mg} \mathrm{O}_{2} \mathrm{~L}^{-1}$ & 5,70 & 1,55 & 6,88 & 0,95 & 5,74 & 1,44 & 6,79 & 0,81 \\
\hline Nitrato & $\mathrm{mg} \mathrm{L}^{-1}$ & 0,195 & 0,057 & 0,435 & 0,092 & 2,51 & 0,702 & 3,960 & 0,863 \\
\hline Fósforo & $\mathrm{mg} \mathrm{L}^{-1}$ & 0,016 & 0,00216 & 0,012 & 0,004 & 0,0240 & 0,0048 & 0,017 & 0,004 \\
\hline Escherichia coli & NMP $100 \mathrm{~mL}^{-1}$ & 118 & & 110 & & 2.790 & & 583 & \\
\hline
\end{tabular}

(M.): média e (D.P.): desvio padrão.

$\mathrm{Na}$ análise dos resultados dos valores obtidos para $\mathrm{pH}$ e temperatura das águas superficiais não apresentaram diferença significativa para P5 e P6 entre os períodos chuvoso e seco e entre os pontos, variando entre 6,2 e 6,8 e 22,6 e $28,8 \stackrel{\circ}{ } \mathrm{C}$, respectivamente. Os resultados obtidos para pH estão de acordo com o estabelecido pela Resolução CONAMA no. 357 (CONAMA, 2005), que determina pH entre 6,0 e 9,0, mas não estabelece limite para temperatura da água.

Comportamento semelhante foi verificado nos parâmetros turbidez e resíduo total, sendo que os valores não apresentaram diferença significativa para P5 e P6 entre os períodos chuvoso e seco e nem entre os pontos, variando entre 0,9 e 25,6 NTU e 4,5 e 54,3 $\mathrm{mg} \mathrm{L}^{-1}$, respectivamente, observado comportamento contrário entre turbidez e resíduos totais, sendo que as maiores médias de turbidez verificadas no período chuvoso e de resíduos totais no seco. Os resultados obtidos para turbidez e resíduo totais estão de acordo com o estabelecido pela Resolução CONAMA no. 357 (CONAMA, 2005), que determina turbidez máxima de 100 NTU e teor máximo de resíduos totais máximo $500 \mathrm{mg} \mathrm{L}^{-1}$.

Resultados semelhantes aos de turbidez e resíduos totais foram obtidos para $\mathrm{DBO}_{5,20}$ e oxigênio dissolvido, não tendo sido observadas diferenças significativas para P5 e P6 entre os períodos chuvoso e seco e nem entre os pontos, variando entre 2,0 e 7,6 $\mathrm{mg} \mathrm{O}_{2} \mathrm{~L}^{-1}$ e 4,3 e 7,8 $\mathrm{mg} \mathrm{O}_{2} \mathrm{~L}^{-1}$, respectivamente, sendo que as maiores médias de $\mathrm{DBO}_{5,20}$ e oxigênio dissolvidos foram verificadas no período seco, sendo que o mesmo comportamento foi observado em resíduos totais. Os resultados médios obtidos para $\mathrm{DBO}_{5,20}$ e oxigênio dissolvido estão de acordo com o estabelecido pela Resolução CONAMA no. 357 (CONAMA, 2005), que determina $\mathrm{DBO}_{5,20}$ máxima de 5,0 $\mathrm{mg} \mathrm{O}_{2} \mathrm{~L}^{-1}$ e teor mínimo de oxigênio dissolvido 5,0 $\mathrm{mg} \mathrm{O}_{2} \mathrm{~L}^{-1}$, apesar de alguns valores de $\mathrm{DBO}_{5,20}$ e de oxigênio dissolvido se apresentarem acima e inferior do estabelecido.

Os dados de nitrato corroboram com os obtidos de resíduos totais, tendo sido observadas as maiores concentrações no período de seca, sugerindo que a redução do volume de águas no período resulta no aumento das concentrações de nitrato e de resíduos totais. Já os resultados de fósforo total estão de acordo com os de turbidez, com os máximos observados no período chuvoso, sugerindo que o carreamento de partículas orgânicas pelas águas das chuvas, resultam em maiores concentrações de fósforo total e turbidez.

É possível concluir que P5, por apresentar uma vegetação mais densa e preservada do que P6, que se encontrava mais desflorestado e que possivelmente foi impactado pelo assoreamento em função da construção do tanque de piscicultura nas mediações, devendo ser por isso a diferença verificada entre P5 e 
P6, sendo que a concentração de nitrato foi 12,9 e 9,1 vezes maior em P6 que P5 para chuva e seca, respectivamente.

Os dados de para Escherichia coli e do cálculo do índice de qualidade corroboram os de nitrato, onde verifica-se que não houve diferença significativa entre os períodos, no entanto ouve diferença para os pontos P5 e P6. Na análise das concentrações de $E$. coli, verifica-se que em P5 a concentração se manteve próxima aos 114 NPM $100 \mathrm{~mL}^{-1}$ nos dois períodos, enquanto para P6 foi de 2.790 e de $593 \mathrm{NPM} 100 \mathrm{~mL}^{-1}$ para o período chuvoso e seco, respectivamente, ou seja, de 4,7 vezes superior à concentração do período chuvoso em comparação com o seco. Somente a concentração observada em P6 para o período chuvoso ficou fora do limite estabelecido pela CONAMA $n^{\circ}$. 357 (CONAMA, 2005), que determina valores máximos 1.000 NPM $100 \mathrm{~mL}^{-1}$.

Os dados de nitrato e Escherichia coli refletem-se nos valores obtidos para índice de qualidade das águas (IQA) do Córrego do Meio, demonstrando uma maior deterioração da qualidade em P6, com IQA atingindo um limite inferior de 58,9 e 61,1\% nos períodos chuvoso e seco, respectivamente, enquanto que P5 apresentou mínimos de 66,8 e 67,1\%, consecutivamente, o que representa uma classificação regular segundo os limites estabelecidos nas faixas de IQA (regular, para valores entre 51 e 70). O máximo apresentado pelo índice foi de 73,3\% para P5 no período chuvoso, o que representa uma classificação das águas considerada boa (boa, valores acima de 71).

\section{CONCLUSÕES}

Nas águas subterrâneas, observou-se maiores concentrações nos períodos de seca para os parâmetros de condutividade elétrica, cor real e aparente, turbidez, sólidos totais, nitrito, nitrato fósforo, dureza total, $\mathrm{DBO}_{5,20}$ e DQO, onde ocorre maior concentração dos compostos orgânicos, ocasionando um aumento nas concentrações dos parâmetros analisados.

A nascente do Córrego do Meio foi classificada através do IQA como Boa e o ponto a Jusante como Regular. Essa classificação inferior se deve aos parâmetros de Escherichia coli e nitrato, que contribuíram para os menores valores de IQA em P6.

Os resultados obtidos inferem que até o presente estudo não apresentou carreamento de poluentes do antigo lixão capaz de impactar na qualidade das águas subterrâneas e superficiais. Portanto, verificou-se que a localização do lixão está em uma área de subsolo impermeável, dificultando a lixiviação de compostos provenientes da disposição inadequada de resíduos sólidos urbanos.

\section{REFERÊNCIAS}

ABRELPE. Associação Brasileira de Empresas de Limpeza Pública e Resíduos Especiais. Panorama 2017. ABRELPE, 2018.

APHA. American Public Health Association. Standard methods for the examination of water and wastewater. 22 ed. Washington: APHA, 2012.

BEHLING, M.. Nutrição, partição de biomassa e crescimento de povoamentos de teca em Tangará da Serra-MT. Tese (Doutorado em Solos e Nutrição de Plantas) - Universidade Federal de Viçosa, Viçosa, 2009.

BRASIL. Plano de Conservação da Bacia do Alto Paraguai. Brasília: Projeto Pantanal, 1997.

BRASIL. Sistema Nacional de Informações sobre Saneamento. Diagnóstico dos Serviços de Água e Esgotos - 
2017. Ministério do Desenvolvimento Regional, 2017a.

BRASIL. Portaria de Consolidação n. 5: Padrão de potabilidade da água. Brasília: Ministério da Saúde, 2017b.

CONAMA. Conselho Nacional do Meio Ambiente. Resolução n. 357: Classificação de águas superficiais. Brasília: Ministério do Meio Ambiente, 2005.

CONAMA. Conselho Nacional do Meio Ambiente. Resolução n. 396: Classificação e enquadramento das águas subterrâneas. Brasília: Ministério do Meio Ambiente, 2008.

CRUZ, J. P.; GARCIA, H. L.; BOECHAT, C. P. S.; CHAGAS, J. C. C.; SMIT, L.; SARAIVA, G. N.; OLIVEIRA, M. L. R.. Growth and yield models for young stands of Tectona grandis in Tangará da Serra, MatoGrosso, Brazil. Revista Arvore, v.32, n.5, p.821-828, 2008. DOI: http://doi.org/10.1590/S010067622008000500006

DALLACORT, R.; MARTINS, J. A.; INOUE, M. H.; FREITAS, P. S. L.; KRAUSE, W.. Agroclimatic aptitude of oil seed plant in the region of Tangará da Serra, Mato Grosso state, Brazil. Revista Ciência Agronômica, v.41, n.3, p.373-379, 2010. DOI: $\underline{\text { https://doi.org/10.1590/S1806-66902010000300008 }}$

FEITOSA, A C. F.; MANOEL FIHO, J.. Hidrogeologia: Conceitos e Aplicações. CPRM - Serviço Geológico do Brasil. Fortaleza: Gráfica LCR, 2008.

GOUVEIA, R. G. L.. Análise da conservação ambiental da bacia hidrográfica rio Queima Pé: Município de Tangará da
Serra/MT. Dissertação (Mestrado em Ambiente e Sistemas de Produção Agrícola) - Universidade do Estado de Mato Grosso, Tangará da Serra, 2013.

IBGE. Instituto Brasileiro de Geografia e Estatística. Censo Demográfico 2010: características da população e dos domicílios: resultados do universo. Rio de Janeiro: IBGE, 2011.

KAPELEWSKA, J.; KOTOWSKA, U.; KARPIŃSKA, J.; ASTEL, A.; ZIELIŃSKI, P.; SUCHTA, J.; ALGRZYM, K.. Water pollution indicators and chemometric expertise for the assessment of the impact of municipal solid waste landfills on groundwater located in their area. Chemical Engineering Journal, v.359, p.790-800, 2019. DOl: http://doi.org/10.1016/j.cej.2018.11.137

MELO, M. T.; QUEIROZ, T. M.; VINAGA, L.; FERREIRA, J. R. S.; MARCHETTO, M.. Variabilidade das classes de água e Índice de Estado Trófico do Riacho Queima Pé. Revista Engenharia na Agricultura - Reveng, v.27, n.1, p.68-79, 2019. DOI: http://doi.org/10.13083/reveng.v27i1.845

MISHRA, S.; TIWARY, D.; OHRI, A.; AGNIHOTRI, A. K.. Impact of Municipal Solid Waste Landfill leachate on groundwater quality in Varanasi, India. Groundwater for Sustainable Development., v.9, 2019. DOI: http://doi.org/10.1016/j.gsd.2019.100230

TANGARÁ DA SERRA. Plano Municipal de Saneamento Básico de Tangará da Serra-MT. Tangará da Serra: Prefeitura Municipal, 2020.

A CBPC - Companhia Brasileira de Produção Científica (CNPJ: 11.221.422/0001-03) detém os direitos materiais desta publicação. Os direitos referem-se à publicação do trabalho em qualquer parte do mundo, incluindo os direitos às renovações, expansões e disseminações da contribuição, bem como outros direitos subsidiários. Todos os trabalhos publicados eletronicamente poderão posteriormente ser publicados em coletâneas impressas sob coordenação da Sustenere Publishing, da Companhia Brasileira de Produção Científica e seus parceiros autorizados. Os (as) autores (as) preservam os direitos autorais, mas não têm permissão para a publicação da contribuição em outro meio, impresso ou digital, em português ou em tradução. 